\title{
Localization of Mobile Nodes in Wireless Networks with Correlated in Time Measurement Noise
}

\author{
Lyudmila Mihaylova, Senior Member, IEEE, Donka Angelova, \\ David R. Bull, Senior Member, IEEE, and Nishan Canagarajah, Member, IEEE
}

\begin{abstract}
Wireless sensor networks are an inherent part of decision making, object tracking, and location awareness systems. This work is focused on simultaneous localization of mobile nodes based on received signal strength indicators (RSSIs) with correlated in time measurement noises. Two approaches to deal with the correlated measurement noises are proposed in the framework of auxiliary particle filtering: with a noise augmented state vector and the second approach implements noise decorrelation. The performance of the two proposed multimodel auxiliary particle filters (MM AUX-PFs) is validated over simulated and real RSSIs and high localization accuracy is demonstrated.
\end{abstract}

Index Terms-Wireless networks, auxiliary particle filtering, localization, correlated measurement noises, multiple model estimation, auxiliary particle filtering.

\section{INTRODUCTION}

$\mathrm{T}$ HE movement patterns of mobile users play an important role in wireless networks in which nodes can move freely within an area. It is essential when nodes perform jointly certain tasks, such as decision making, sensor data fusion, object tracking [1], [2], [3], [4], [5] to localize the node positions, and movement [6], [7], [8], as the transmitter range is generally fairly small with respect to the size of the area. Other motivating applications include monitoring of large geographical areas, such as for wildlife tracking [9], monitoring of buildings, production processes, and warehouses [7]. Apart from the changeable network topology, the need of communications between the nodes under limited resources (energy and bandwidth) and the need of processing noisy data and overcoming losses pose additional challenges.

There is a great deal of methods for localization (see, e.g., the surveys [6], [7], [10], [11]) between which the range-based [7], [12], [13] methods are widely used. They rely on the distances between nodes and are usually evaluated using received signal strengths, signal time-of-arrivals, time difference of arrivals, or angle-of-arrivals, and they vary in their complexity and accuracy. The range-based techniques can be divided into radio frequency (RF) ranging and acoustic

- L. Mihaylova is with Lancaster University, InfoLab21, South Drive, Lancaster LA1 4WA, United Kingdom.

E-mail: mila.mihaylova@lancaster.ac.uk.

- D. Angelova is with the Bulgarian Academy of Sciences, Central Laboratory for Parallel Processing, Acad. G. Bonchev Str., Bl. 25 A, 1113 Sofia, Bulgaria. E-mail: donka@bas.bg.

- D.R. Bull and N. Canagarajah are with the University of Bristol, Merchant Venturers Building, Woodland Road, Bristol BS8 1UB, United Kingdom. E-mail: dave.bull@bristol.ac.uk, nishan.canagarajah@lancaster.ac.uk.

Manuscript received 24 Nov. 2008; revised 18 Dec. 2009; accepted 25 Apr. 2010; published online 1 Aug. 2010.

For information on obtaining reprints of this article, please send e-mail to: tmc@computer.org, and reference IEEECS Log Number TMC-2008-11-0469. Digital Object Identifier no. 10.1109/TMC.2010.132. ranging. The radio frequency ranging relies on the premise that by measuring the received signal strength, a receiver can determine its distance to a transmitter. Another class of ranging schemes measures the time difference of arrival of acoustic and ultrasonic signals [12], [14].

While range-based algorithms need point-to-point distance estimation or angle estimation for positioning, rangefree [15] algorithms do not require this information. Another classification subdivides the approaches to mobile nodes localization in wireless networks to indoor and outdoor environment [11].

From the point of view of the methods employed, a number of localization techniques rely on Extended Kalman filters [16], [17], Monte Carlo methods [18], [19], including nonparametric belief propagation [20], and on the knowledge of the connectivity between the nodes. Communications between nodes during the localization process are reduced to minimum due to energy and bandwidth constraints. In [21] and [22], multiple model particle filtering techniques for mobility tracking of users in cellular networks are developed. A particle filter and a RaoBlackwellized particle filter are presented and their performance is compared with an Extended Kalman filter over simulated and real data from base stations. In [23], [24], an auxiliary multiple model particle filter $(\mathrm{PF})$ is proposed for bearings-only tracking problems. In [23], a deterministic splitting of each particle into several offsprings is performed for maneuvering target tracking, each offspring representing a different target maneuver. In [25], an auxiliary PF is designed for target tracking in binary sensor networks. When the measurements are collinear, a simulated annealing approach, such as the proposed in [26], can cope with these ambiguities.

Most of the aforementioned works, however, do not take into account the correlation of the measurement noise which can deteriorate significantly the localization accuracy. One of 
the most common correlation models for the slow shadow fading component is proposed by Gudmunsson [27]. The model of Gudmunsson consists of a decreasing autocorrelation function. The same first order autoregressive correlation model is used in [28], jointly with a Kalman filter aimed to estimate the correlation coefficient of the shadowing component of the measurements. The shadow fading correlation properties are studied also in [29].

In our work, we propose a solution to the selflocalization problem of mobile nodes by taking into account the temporal correlation in the measurement noise. The added values and the innovative aspects of this paper as compared with previous investigations consist in the approach for localization of a single or several mobile nodes in wireless sensor networks by means of a multiple model auxiliary particle filter. Its performance is validated on simulated and real received signal strength indicators, over scenarios with several nodes and a single mobile node, respectively. Similarly to [27], the correlated noise is modeled by a first order autoregressive model. Two approaches, to deal with the correlated measurement noises, are proposed based on ideas inspired from the tracking literature. The first one augments the state vector with the measurement noise, and the second approach implements a noise decorrelation by introducing the socalled "differenced measurement" [30]. Two algorithms are designed and implemented in the MM AUX-PF framework. The aim is to explore and compare the localization performance of the proposed MM AUX-PF with and without taking into account the noise correlation.

In the considered formulation of the problem, node mobility is modeled as a linear system driven by a discrete-time command Markov process, whereas the measurement models are nonlinear and necessitate a reliable nonlinear estimation method. Due to the fact that the control process of the mobile nodes is unknown, node mobility is modeled with multiple acceleration modes (regimes). The proposed nonlinear estimation techniques can incorporate physical constraints and possibly communications among frequently maneuvering mobile nodes in the form of additional measurements.

The remaining part of the paper is organized as follows: Section 2 formulates the considered problem for localization of mobile nodes and describes the mobility and observation models. A multiple model auxiliary particle filter (MM AUX-PF) for mobile nodes self-localization is designed in Section 3. Performance evaluation of the proposed filter is presented in Section 4 over simulated and real data. Finally, Section 5 discusses the results and outlines open issues for future research.

\section{Localization of Mobile Nodes}

Consider the two-dimensional problem of simultaneous localization of $n$ mobile nodes [7]. The vector $\left\{\left(x_{1}, y_{1}\right)\right.$, $\left.\left(x_{2}, y_{2}\right), \ldots,\left(x_{n}, y_{n}\right)\right\}$ of positions of the mobile nodes is estimated given $n_{r}$ reference (anchor) nodes with known coordinates $\left\{\left(x_{n+1}, y_{n+1}\right),\left(x_{n+2}, y_{n+2}\right), \ldots,\left(x_{n+n_{r}}, y_{n+n_{r}}\right)\right\}$ and pairwise measurements $\left\{z_{i j}\right\}$, where $z_{i j}$ is a measurement between devices $i$ and $j$. The reference nodes can obtain their coordinates in the network (through some external means such as a Global Positioning System (GPS)).
Apart from their positions, the mobile nodes estimate their speeds and accelerations. This includes applications in which each sensor is equipped with a wireless transceiver and the distance between sensor locations is estimated by received signal strength indicator (RSSI) measurements or time delay of arrivals between sensor locations.

\subsection{Motion Model of the Mobile Nodes}

Different state mobility models are previously developed for cellular wireless networks such as random walk and pursue mobility models [31] and Singer-type models [6], [32], [33], [34]. Most of the models suggested in the target tracking literature [6], [35], [36] are also applicable for mobile nodes localization. In this paper, we choose a discrete-time Singer-type model [37] because it characterizes the correlated accelerations of the mobile as a time correlated process and allows for accurate prediction of the position, speed, and acceleration of mobile nodes. This higher order model affords decreasing the estimation error [6], although mobility models that do not comprise the acceleration can be used too.

The state of each moving node at time instant $k$ is defined by the vector $x_{k}=\left(x_{k}, \dot{x}_{k}, \ddot{x}_{k}, y_{k}, \dot{y}_{k}, \ddot{y}_{k}\right)^{\prime}$, where $x_{k}$ and $y_{k}$ specify the position, $\dot{x}_{k}$ and $\dot{y}_{k}$ specify the speed, $\ddot{x}_{k}$ and $\ddot{y}_{k}$ are, respectively, the acceleration in $x$ and $y$ directions in the two-dimensional plane, and ' denotes the transpose operation. The motion of each mobile node can be described by the following Singer model [37], [38]:

$$
\boldsymbol{x}_{k}=\boldsymbol{A}(T, \alpha) \boldsymbol{x}_{k-1}+\boldsymbol{B}_{u}(T) \boldsymbol{u}_{k}+\boldsymbol{B}_{w}(T) \boldsymbol{w}_{k},
$$

where $\boldsymbol{u}_{k}=\left(u_{x, k}, u_{y, k}\right)^{\prime}$ is a discrete-time command process, and the respective matrices from (1) are in the form

$$
\begin{gathered}
A(T, \alpha)=\left(\begin{array}{cc}
\tilde{A} & 0_{3 \times 3} \\
\mathbf{0}_{3 \times 3} & \tilde{\boldsymbol{A}}
\end{array}\right), B_{i}(T)=\left(\begin{array}{cc}
\tilde{B}_{i} & 0_{3 \times 1} \\
\mathbf{0}_{3 \times 1} & \tilde{\boldsymbol{B}}_{i}
\end{array}\right), \\
\tilde{\boldsymbol{A}}=\left(\begin{array}{ccc}
1 & T & T^{2} / 2 \\
0 & 1 & T \\
0 & 0 & \alpha
\end{array}\right), \tilde{\boldsymbol{B}}_{u}=\left(\begin{array}{c}
T^{2} / 2 \\
T \\
0
\end{array}\right), \tilde{\boldsymbol{B}}_{w}=\left(\begin{array}{c}
T^{2} / 2 \\
T \\
1
\end{array}\right) .
\end{gathered}
$$

The subscript $i$ in the matrix $B(T)$ in (2) stands for $u$ or $w$, respectively. The random process $\boldsymbol{w}_{k}$ is a $2 \times 1$ vector and $T$ is the discretization period. The parameter $\alpha$ is the reciprocal of the maneuver time constant, and thus, depends on how long the maneuver lasts. The process noise $w_{k}$ is a white sequence, with covariance matrix $E\left[\boldsymbol{w} \boldsymbol{w}^{\prime}\right]=\boldsymbol{Q}=\sigma_{w}^{2} I$, where $E[$.$] is the mathematical expecta-$ tion operation, $I$ denotes the unit matrix, and $\sigma_{w}$ is the standard deviation.

The unknown command processes $u_{x, k}$ and $u_{y, k}$ take values from a set of acceleration levels $\mathcal{M}_{x}$ and $\mathcal{M}_{y}$. The process $u_{k}$ takes values from the set $\mathbb{M}=\mathcal{M}_{x} \times \mathcal{M}_{y} \triangleq\left\{\boldsymbol{u}_{1}, \ldots, \boldsymbol{u}_{r}\right\}$. Let $\mathbb{S} \triangleq\{1,2, \ldots, r\}$ denote the set of models and let $m_{k} \in \mathbb{S}$ be the regime variable, modeled as a first order Markov chain with transition probabilities $\Pi_{i j}=P\left(m_{k}=j \mid m_{k-1}=i\right), i, j=$ $1, \ldots, r$, and initial probability distribution $\tilde{\mu}_{i, 0}=P_{0}\{m=$ $\left.m_{i}\right\}$ for $m_{i} \in \mathbb{S}$ such that $\tilde{\mu}_{i, 0} \geq 0$ and $\sum_{i=1}^{r} \tilde{\mu}_{i, 0}=1$.

\subsection{Observation Model}

In wireless networks, the distance between mobile and reference (anchor) nodes can be inferred from RSSIs or pilot signals of nodes. The RSSI $z_{\ell j, k}$ received at the mobile node 
$N_{\ell}$ with coordinates $\left(x_{\ell, k}, y_{\ell, k}\right)$ at time $k$, after being transmitted from the node $N_{j}$ with coordinates $\left(x_{j, k}, y_{j, k}\right)$, propagates as follows [6], [27], [39]:

$$
z_{\ell j, k}=\kappa_{j}-10 \gamma \log _{10}\left(d_{\ell j, k}\right)+v_{\ell j, k},
$$

where $\kappa_{j}$ is a constant depending on the transmission power, wavelength, and gain of node $N_{j}, \gamma$ is the slope index (according to [27] $\gamma=3.3$ for suburban environment and $\gamma=5$ for microcells in urban environment), $v_{\ell j, k}$ is the logarithm of the shadowing component, which is usually correlated in time, and $d_{\ell j, k}$ is the distance between nodes $N_{\ell}$ and $N_{j}$

$$
d_{\ell j, k}=\sqrt{\left(x_{\ell, k}-x_{j, k}\right)^{2}+\left(y_{\ell, k}-y_{j, k}\right)^{2}} .
$$

All mobile nodes in the group send their pilot signals to the reference nodes. In order to locate a single mobile node in the two-dimensional plane, the three largest RSSIs to reference neighboring nodes are necessary to enable triangulation. The measurement equation can be written in the form

$$
z_{k} \triangleq\left(z_{\ell 1, k}, z_{\ell 2, k}, z_{\ell 3, k}\right)^{\prime}=\boldsymbol{h}\left(\boldsymbol{x}_{k}\right)+\boldsymbol{v}_{k},
$$

where $\boldsymbol{h}\left(\boldsymbol{x}_{k}\right)=\left(h_{\ell 1, k}, h_{\ell 2, k}, h_{\ell 3, k}\right)^{\prime}$ is a nonlinear function, with components $h_{\ell j, k}=\kappa_{j}-10 \eta \log _{10}\left(d_{\ell j, k}\right), j=1,2,3$, $z_{k} \in \mathbb{R}^{n_{z}}$, and $n_{z}=3$. The noise $\boldsymbol{v}_{k}=\left(v_{1, k}, v_{2, k}, v_{3, k}\right)^{\prime}$, $\boldsymbol{v}_{k} \in \mathbb{R}^{n_{z}}$, with covariance $E\left[\boldsymbol{v}_{k} \boldsymbol{v}_{k}^{\prime}\right]=\boldsymbol{R}_{k}$, characterizes the shadowing components.

In the general case with $\ell=1, \ldots n$ mobile nodes and $j=1, \ldots n_{r}$ reference nodes, the overall observation vector will contain $L=n * n_{r}$ number of measurements.

\subsection{Correlated in Time Measurement Noise}

In urban and suburban environment, the autocorrelation function of the measurement noise (shadowing component) $v_{\ell j, k}$ from (4) can be modeled with the relation [27], [28]

$$
c_{v}(\tau)=\sigma_{v}^{2} \exp \left\{-\mathrm{v}|\tau| / D_{c}\right\},
$$

where $\tau$ is the time lag, $\sigma_{v}$ denotes the standard deviation of the shadowing process, $D_{c}$ is the effective correlation distance, which is of key importance in a wireless environment, and $\mathrm{v}$ is the velocity of the mobile node. In [27], it is shown that

$$
D_{c}=-\frac{d_{\ell j, k}}{\ln \left(\epsilon_{D}\right)} \geq 0
$$

where $\epsilon_{D}$ is the correlation coefficient of the shadow process between two mobile nodes separated by the distance $d_{\ell j, k}$. Usually, $D_{c}$ is in the range between 10 meters in urban environment and 500 meters in suburban environment. The value of the shadow standard deviation $\sigma_{v}$ varies dependent on the environment and in suburban areas is typically $8 \mathrm{~dB}$ [27], [28], whereas in urban environments, it is roughly $4 \mathrm{~dB}$.

The shadow process can be modeled by a first order autoregressive model (AR) [27], [28]

$$
v_{\ell j, k}=a v_{\ell j, k-1}+\phi_{k},
$$

where $\phi_{k}$ is a zero mean white Gaussian process with variance $\sigma_{\phi}^{2}=\left(1-a^{2}\right) \sigma_{v}^{2}$. The coefficient $a$ is given by

$$
a=\exp \left(-\mathrm{v} T / D_{c}\right),
$$

where $T$ is the measurement sampling period.

It is assumed that the AR model parameters (correlation coefficient and variance) are known and have typical values for urban and suburban environments. These parameters are experimentally obtained by different authors for urban and suburban environment, e.g., in [27], [28], [40].

\subsection{General Motion and Observation Models for Simultaneous Localization of Several Mobile Nodes}

A combined state vector $X_{k}=\left\{x_{1, k}^{\prime}, \ldots, x_{n, k}^{\prime},\right\}$ is composed and all states of the mobile nodes are simultaneously estimated. The motion models (1)-(3) can be generalized to the form

$$
X_{k}=f\left(X_{k-1}, \mathrm{M}_{k}, U_{k}, W_{k}\right),
$$

where $X_{k} \in \mathbb{R}^{n * n_{x}}$ is the combined system base state vector, $U_{k} \in \mathbb{R}^{n * n_{u}}$ specifies the command processes for all mobile nodes, and the modal (discrete) state $\mathrm{M}_{k} \in \mathbb{S}$ of the different system modes (regimes). The dimension of the combined system noise vector is $W_{k} \in \mathbb{R}^{n * n_{w}}$.

The measurement equation (6) can be generalized to

$$
\begin{aligned}
& Z_{k}=h\left(X_{k}\right)+V_{k}, \\
& V_{k}=a V_{k-1}+\Phi_{k},
\end{aligned}
$$

where $Z_{k} \in \mathbb{R}^{n * n_{z}}$ is a generalized measurement vector, and the generalized noise vector $V_{k} \in \mathbb{R}^{n * n_{z}}$ characterizes the correlated in time shadowing components; $\Phi_{k}$ is a $\left(n * n_{z}\right)$ dimensional white noise with covariance matrix $E\left[\Phi_{k} \boldsymbol{\Phi}_{k}^{\prime}\right]=$ $\sigma_{\phi}^{2} I$ and $I$ denotes the identity matrix.

Equations (11)-(13) constitute the whole model for the motion of the mobile nodes and observations with a correlated in time noise.

\section{A Multiple Model Auxiliary Particle FILTERING FOR LOCALIZATION}

\subsection{The Particle Filtering Framework}

Within particle filtering, the localization of mobile nodes reduces to approximation of the state probability density function (PDF) given a sequence of measurements. According to the Bayes' rule, the filtering PDF $p\left(X_{k} \mid Z_{1: k}\right)$ of the state vector $X_{k} \in \mathbb{R}^{n * n_{x}}$ given a sequence of sensor measurements $Z_{1: k}$ up to time $k$ may be written as

$$
p\left(\boldsymbol{X}_{k} \mid Z_{1: k}\right)=\frac{p\left(\boldsymbol{Z}_{k} \mid \boldsymbol{X}_{k}\right) p\left(\boldsymbol{X}_{k} \mid \boldsymbol{Z}_{1: k-1}\right)}{p\left(\boldsymbol{Z}_{k} \mid \boldsymbol{Z}_{1: k-1},\right)},
$$

where $\left(Z_{k} \mid Z_{1: k-1}\right)$ is the normalizing constant. The state predictive distribution is given by the Chapman-Kolmogorov equation

$$
p\left(\boldsymbol{X}_{k} \mid \boldsymbol{Z}_{1: k-1}\right)=\int_{\mathbb{R}^{n * n_{x}}} p\left(\boldsymbol{X}_{k} \mid \boldsymbol{X}_{k-1}\right) p\left(\boldsymbol{X}_{k-1} \mid Z_{1: k-1}\right) d \boldsymbol{X}_{k} .
$$

The evaluation of the right-hand side of (14) involves integration which can be avoided in the particle filtering 
approach [41] by approximating the posterior PDF $p\left(\boldsymbol{X}_{k} \mid Z_{1: k}\right)$ with a set of particles $\boldsymbol{X}_{0: k}^{(i)}, i=1, \ldots, N$, and their corresponding weights $w_{k}^{(i)}$. Then, the posterior density can be written as follows:

$$
p\left(X_{0: k} \mid Z_{1: k}\right)=\sum_{i=1}^{N} w_{k}^{(i)} \delta\left(X_{0: k}-X_{0: k}^{(i)}\right)
$$

where $\delta($.$) is the Dirac delta function, and the weights are$ normalized such that $\sum_{i} w^{(i)}=1$.

Each pair $\left\{\boldsymbol{X}_{0: k}^{(i)}, w_{k}^{(i)}\right\}$ characterizes the belief that the object is in state $X_{0: k}^{(i)}$. An estimate of the variable of interest is obtained by the weighted sum of particles. Two major stages can be distinguished: prediction and update. During prediction, each particle is modified according to the state model, including the addition of random noise in order to simulate the effect of the noise on the state. In the update stage, each particle's weight is reevaluated based on the new data. A resampling procedure introduces variety in the particles by eliminating those with small weights and replicating the particles with larger weights such that the approximation in (16) still holds. The residual resampling algorithm [42], [43] is applied here. This is a two-step process making use of sampling-importance-resampling scheme.

Since the command process of the mobile nodes is unknown, an MM AUX-PF is designed for localization of the mobile nodes. Given the set $\mathbb{M}$ covering well the possible command values, the unknown commands are supposed to evolve as a first order Markov chain with transition probability matrix $\Pi$. The particles for the base state are generated from the transition prior, according to (11)-(12) (where the motion model for each mobile is given by (1)-(3)).

\subsection{Auxiliary Multiple Model Particle Filtering for Localization}

The auxiliary Sampling Importance Resampling (SIR) PF was introduced by Pitt and Shephard [44]. The auxiliary PF draws particles from an importance function which is close as possible to the optimal one. The auxiliary PF introduces an importance function $q\left(X_{k}, i^{(j)}\right)_{i=1}^{N}$, where $i^{(j)}$ refers to the index of the particle at $k-1$. The filter obtains samples from the joint density $p\left(X_{k}, i \mid Z_{1: k}\right)$, and then, omits the index $i$ in the pair $\left(X_{k}, i\right)$ to produce a sample $\left\{X_{k}^{(i)}\right\}_{i=1}^{N}$ from the marginalized density $p\left(X_{k} \mid Z_{1: k}\right)$. The importance density that generates the sample $\left\{X_{k}^{(i)}\right\}_{i=1}^{N}$ is defined to satisfy the relation [41]

$$
q\left(\boldsymbol{X}_{k}, i \mid \boldsymbol{Z}_{1: k}\right) \propto p\left(\boldsymbol{Z}_{k} \mid \boldsymbol{\mu}_{k}^{(i)}\right) p\left(\boldsymbol{X}_{k} \mid \boldsymbol{X}_{k-1}^{(i)}\right) w_{k-1}^{(i)},
$$

where $\boldsymbol{\mu}_{k}^{(i)}$ is some characteristic of $\boldsymbol{X}_{k}$ given $\boldsymbol{X}_{k-1}^{(i)}$.

The selection of the most promising particles is carried out by sampling from a multinomial distribution where the number of possible outcomes is $N^{\text {out }}$. The auxiliary PF [44] resamples the predicted particles to select which particles to use in the prediction and measurement update.

For the purposes of mobile node localization, we propose an auxiliary MM PF. The MM AUX-PF represents the PDF $p\left(\boldsymbol{X}_{k}, i, \mathrm{M}_{k} \mid \boldsymbol{Z}_{1: k}\right)$, where $i$ refers to the $i$ th particle at $k-1$.
After marginalization, the representation of $p\left(X_{k} \mid Z_{1: k}\right)$ can be obtained.

Similarly to [24], the joint probability density $p\left(X_{k}, i\right.$, $\mathrm{M}_{k} \mid Z_{1: k}$ ) can be written using the Bayesian rule as:

$$
\begin{aligned}
& p\left(\boldsymbol{X}_{k}, i, \mathrm{M}_{k} \mid \boldsymbol{Z}_{1: k}\right) \propto p\left(\boldsymbol{Z}_{k} \mid \boldsymbol{X}_{k}\right) p\left(\boldsymbol{X}_{k}, i, \mathrm{M}_{k} \mid \boldsymbol{Z}_{1: k-1}\right) \\
& \quad=p\left(\boldsymbol{Z}_{k} \mid \boldsymbol{X}_{k}\right) p\left(\boldsymbol{X}_{k} \mid \boldsymbol{X}_{k-1}^{(i)}, \mathrm{M}_{k}\right) p\left(\mathrm{M}_{k} \mid \mathrm{M}_{k-1}^{(i)}\right) w_{k-1}^{(i)},
\end{aligned}
$$

where $p\left(\mathrm{M}_{k} \mid \mathrm{M}_{k-1}\right)$ is an element of the transition probability matrix $\Pi$. Since sampling directly from $p\left(X_{k}, i, \mathrm{M}_{k} \mid Z_{1: k}\right)$ is difficult, the following importance function $q\left(X_{k}, i, \mathrm{M}_{k} \mid Z_{1: k}\right)$ is introduced:

$$
\begin{aligned}
q\left(\boldsymbol{X}_{k}, i, \mathrm{M}_{k} \mid \boldsymbol{Z}_{1: k}\right) \propto & p\left(\boldsymbol{Z}_{k} \mid \boldsymbol{\mu}_{k}^{(i)}\left(\mathrm{M}_{k}\right)\right) p\left(\boldsymbol{X}_{k} \mid \boldsymbol{X}_{k-1}^{(i)}, \mathrm{M}_{k}\right) \\
& * p\left(\mathrm{M}_{k} \mid \mathrm{M}_{k-1}^{(i)}\right) w_{k-1}^{(i)},
\end{aligned}
$$

where

$$
\boldsymbol{\mu}_{k}^{(i)}\left(\mathrm{M}_{k}\right)=E\left(X_{k} \mid X_{k-1}^{(i)}, \mathrm{M}_{k}\right) .
$$

The importance density $q\left(X_{k}, i, \mathrm{M}_{k} \mid Z_{1: k}\right)$ differs from (18) only in the first factor. Marginalization over $X_{k}$ yields

$$
q\left(i, \mathrm{M}_{k} \mid \boldsymbol{Z}_{k}\right) \propto p\left(\boldsymbol{Z}_{k} \mid \boldsymbol{\mu}_{k}^{(i)}\left(\mathrm{M}_{k}\right)\right) p\left(\mathrm{M}_{k} \mid \mathrm{M}_{k-1}^{(i)}\right) \cdot w_{k-1}^{(i)} .
$$

By using (21), a random sample from the density $q\left(X_{k}, i, \mathrm{M}_{k} \mid Z_{1: k}\right)$ can be obtained as follows: First, a sample $\left\{i^{(j)}, \mathrm{M}_{k}^{(j)}\right\}_{j=1}^{N}$ is drawn from the multinomial distribution $q\left(i, \mathrm{M}_{k} \mid Z_{1: k}\right),(21)$, by splitting each of the $N$ particles at $k-1$ into $r$ groups. Each of the $N * r$ particles is assigned a weight proportional to the right-hand side of (21). Next, a sample $\left\{\boldsymbol{X}_{k}^{(j)}\right\}_{j=1}^{N}$ from the joint density $q\left(\boldsymbol{X}_{k}, i, \mathrm{M}_{k} \mid Z_{1: k}\right)$ is generated from $p\left(X_{k} \mid X_{k-1}^{\left(i^{j}\right)}, \mathrm{M}_{k}^{(j)}\right)$. To use the samples $\left\{\boldsymbol{X}_{k}^{(j)}, i^{j}, \mathrm{M}_{k}^{(j)}\right\}_{j=1}^{N}$ to characterize the density $p\left(X_{k}, i, \mathrm{M}_{k} \mid Z_{1: k}\right)$, we attach to each particle the weight

$$
w_{k}^{(j)}=\frac{p\left(Z_{k} \mid X_{k}^{(j)}\right)}{p\left(Z_{k} \mid \mu_{k}^{\left(i^{j}\right)}\left(\mathrm{M}_{k}\right)\right)},
$$

which represents the ratio of (19) and (18). By omitting the $\left\{i^{(j)}, \mathrm{M}_{k}^{(j)}\right\}$ components from the triplet sample $\left\{X_{k}^{(j)}, i^{(j)}\right.$, $\left.\mathrm{M}_{k}^{(j)}\right\}_{j=1}^{N}$, we have representation of the marginalized density $p\left(X_{k} \mid Z_{1: k}\right)$, i.e.,

$$
p\left(\boldsymbol{X}_{k} \mid \boldsymbol{Z}_{1: k}\right) \approx \sum_{j=1}^{N} w_{k}^{(j)} \delta\left(\boldsymbol{X}_{k}-\boldsymbol{X}_{k}^{(j)}\right) .
$$

The conditional mean $\mu_{k}^{(i)}\left(\mathrm{M}_{k}^{(i)}\right)$ for each particle in the MM AUX-PF comprises the mean vectors of all mobile nodes. The following deterministic mobility equation is used to calculate the mean for each mobile node:

$$
\boldsymbol{x}_{k}=\boldsymbol{A}(T, \alpha) \boldsymbol{x}_{k-1}+\boldsymbol{B}_{u}(T) \boldsymbol{u}_{k} .
$$

The whole MM AUX-PF for mobile nodes localization is presented as Algorithm 1. The MM AUX-PF takes into account speed constraints, i.e., the speed of each mobile node cannot exceed the maximum value $V_{\max }$. Finally, resampling is performed only when the efficient number of particles $N_{e f f}$ is smaller than a given threshold $N_{\text {thresh }}$. 
Algorithm 1. A multiple model auxiliary PF for mobile nodes localization.

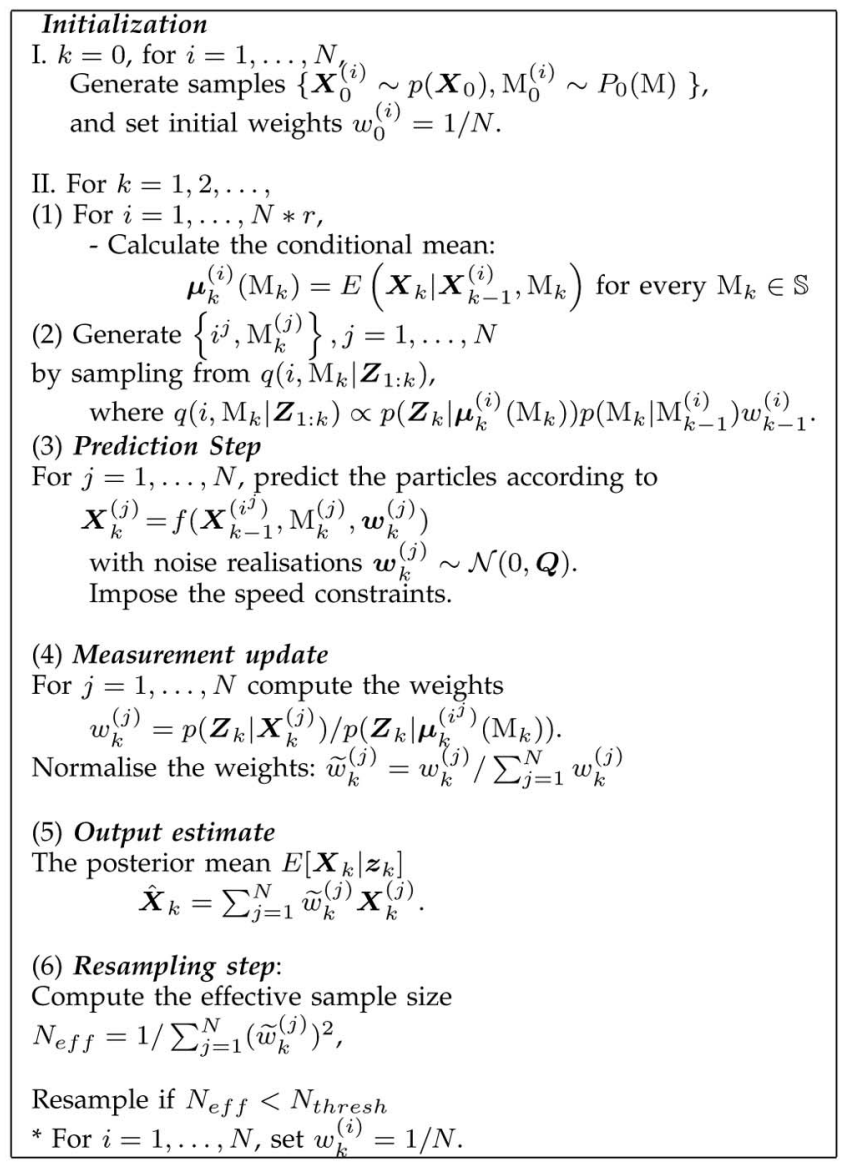

\subsection{Two Approaches to Deal with the Time Correlated Measurement Noise}

A natural solution to overcome the problem with correlated measurement noise is to augment the mobile state $x_{k}$ with the noise $v_{k}$. Then, the localization algorithm (MM AUX-PF) described in Section 3.2 can be applied to the whole augmented state vector of size $n *\left(n_{x}+n_{z}\right)$ (comprising the state vectors of the mobile nodes and measurement noise). This algorithm with the state vector augmented with the correlated noise is referred to as an MM-AUX PF with augmented state (AS).

Another decorrelation technique introduces the following "artificial measurement": $\bar{z}_{k}=z_{k}-a z_{k-1}$. The measurement equation can then be written in the form

$$
\begin{aligned}
\bar{z}_{k} & =z_{k}-a z_{k-1} \\
& =\boldsymbol{h}\left(\boldsymbol{x}_{k}\right)+\boldsymbol{v}_{k}-a\left[\boldsymbol{h}\left(\boldsymbol{x}_{k-1}\right)+\boldsymbol{v}_{k-1}\right] \\
& =\boldsymbol{h}\left(\boldsymbol{x}_{k}\right)-a \boldsymbol{h}\left(\boldsymbol{x}_{k-1}\right)+\overline{\boldsymbol{v}}_{k} .
\end{aligned}
$$

The noise $\overline{\boldsymbol{v}}_{k}=\boldsymbol{v}_{k}-a \boldsymbol{v}_{k-1}$ in the new measurement equation is white but correlated with the process noise. The cross correlation between two noise sequences can be eliminated by a procedure, given in [35]. In most practical algorithms, this cross correlation is omitted due to the little performance degradation. Thus, the measurement equation in the case of one mobile node can be modified to

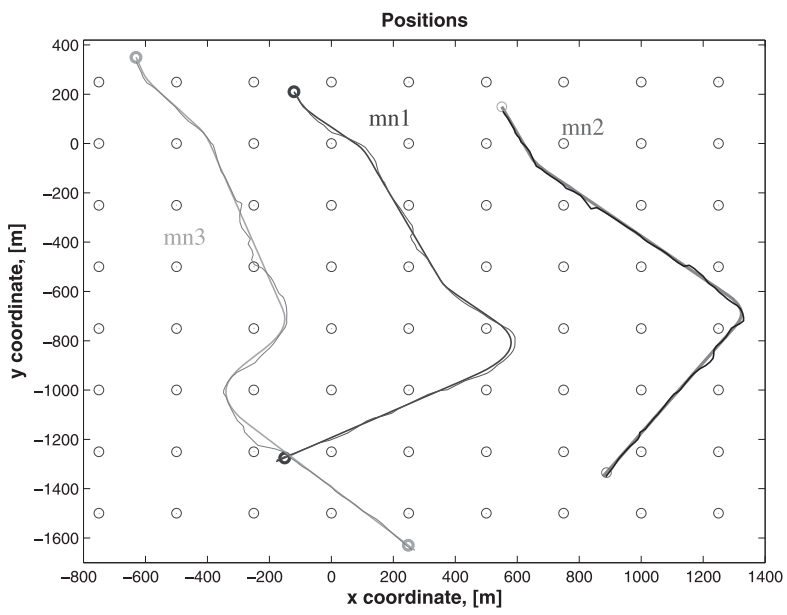

Fig. 1. Testing scenario 1: three mobile nodes ( $\mathrm{mn} 1, \mathrm{mn} 2$, and $\mathrm{mn} 3$ ) moving in an area covered with a wireless sensor network. The sensors are uniformly deployed and form a rectangular grid.

$$
\bar{z}_{k}=\bar{h}\left(x_{k}\right)+\overline{\boldsymbol{v}}_{k},
$$

where $\bar{h}\left(\boldsymbol{x}_{k}\right)=\boldsymbol{h}\left(\boldsymbol{x}_{k}\right)-a \boldsymbol{h}\left(\boldsymbol{x}_{k-1}\right)$.

The MM-AUX PF algorithm with decorrelation is referred to as with an artificial measurement (AM).

\section{Performance Evaluation}

Two cases have been investigated: for urban and suburban environment. In suburban environment, the correlation coefficient of the shadow process can be regarded as a constant for a wide range of velocities of the mobile [28]. Typical values of the correlation coefficient and shadow process are assumed, as suggested in the literature [27].

\subsection{Results with Simulated Data}

\subsubsection{Testing Scenario 1}

A sensor deployment architecture is considered, similar to the presented in [25]. Three mobile nodes are moving in an urban area well covered with a wireless sensor network (Fig. 1). Each mobile node can measure the RSSI to each of the reference nodes, but only the three RSSIs with the highest strength are used for localization.

The MM AUX-PF AS is run for estimating the augmented state vector, consisting of three individual mobile state vectors. Fig. 1 presents the actual and estimated trajectories of the three mobile nodes. The actual speed of the mobiles is shown in Fig. 2.

The parameters of the individual state vector initial distribution $\boldsymbol{x}_{i, 0} \sim \mathcal{N}\left(\boldsymbol{m}_{i, 0}, \boldsymbol{P}_{i, 0}\right)$ are selected as follows:

$$
\begin{aligned}
\boldsymbol{P}_{i, 0} & =\operatorname{diag}\left\{\boldsymbol{P}_{\mathrm{x}, 0}, \boldsymbol{P}_{\mathrm{y}, 0}\right\}, P_{x, 0}=P_{y, 0} \\
& =\operatorname{diag}\left\{30[\mathrm{~m}]^{2}, 1[\mathrm{~m} / \mathrm{s}]^{2}, 0.5\left[\mathrm{~m} / \mathrm{s}^{2}\right]^{2}\right\}, i=1,2,3,
\end{aligned}
$$

and $m_{i, 0}$ contains the exact initial node states. Initial mode probabilities are $\tilde{\mu}_{1,0}=0.8$ and $\tilde{\mu}_{i, 0}=0.05$ for $i=2, \ldots, 5$. The transition probability matrix $\Pi$ has the following diagonal elements: $\Pi_{11}=0.9, \Pi_{i i}=0.7, i=2, \ldots, 5$ and the off-diagonal elements (e.g., $\Pi_{i, 1}=0.025, i=2, \ldots, 5$, $\left.\Pi_{i, 2}=0.07, i=3, \ldots, 5\right)$ are chosen equal in each row to guarantee that the sum in each row is equal to one. The noise correlation coefficient was assumed to be equal to a 


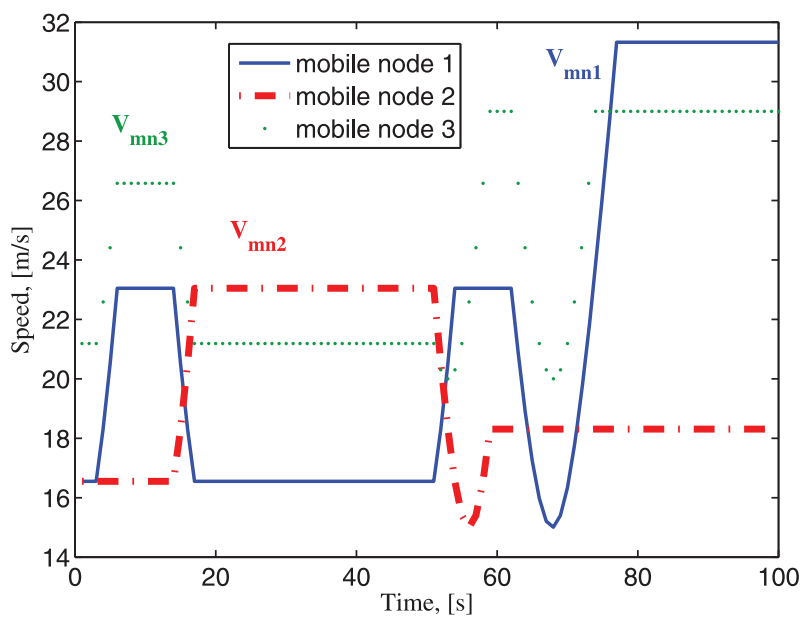

Fig. 2. Actual speeds of the three maneuvering mobile nodes.

TABLE 1

Simulation Parameters for Urban Environment

\begin{tabular}{|c|c|}
\hline Discretisation time step $T$ & $1.0[\mathrm{~s}]$ \\
\hline Correlation coefficient $a$ & 0.25 \\
\hline Path loss index $\gamma$ & 5 \\
\hline Transmission power $\kappa$ & 30 \\
\hline Variance $\sigma_{w}^{2}$ of the noise $\boldsymbol{w}_{k}$ in $(1)$ & $0.5^{2}\left[\mathrm{~m} / \mathrm{s}^{2}\right]^{2}$ \\
\hline Maximum speed $V_{\max }$ & $45[\mathrm{~m} / \mathrm{s}]$ \\
\hline Number of particles of the MM AUX-PF & $N=500$ \\
\hline Threshold for resampling & $N_{t h r e s h}=N / 10$ \\
\hline Number of Monte Carlo runs & $N_{m c}=50$ \\
\hline Variance $\sigma_{v}^{2}$ of the measurement noise $v_{k}$ & $4^{2}[\mathrm{~dB}]^{2}$ \\
\hline
\end{tabular}

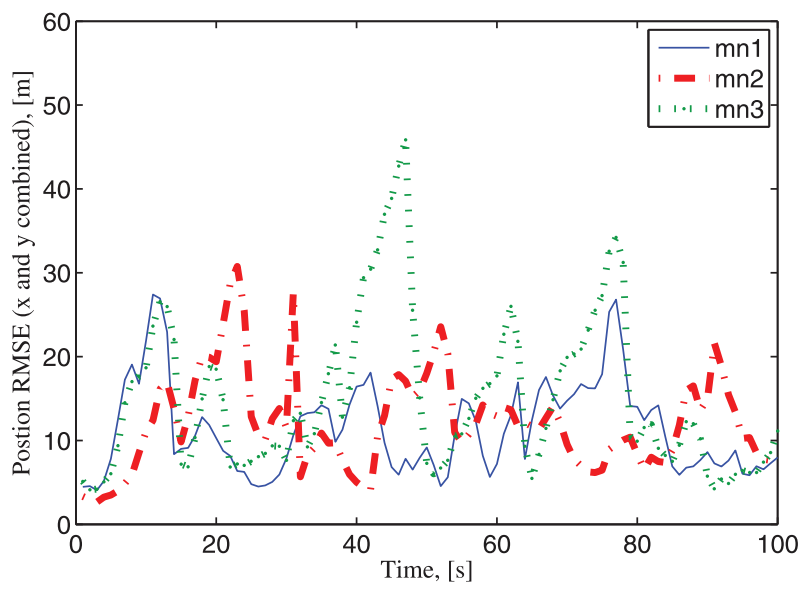

Fig. 3. Results for the position RMSE obtained with the MM AUX-PF with an augmented state vector.

typical value for urban environment, 0.25 . The correlated measurement noises are generated by means of Cholesky factorization.

The other parameters of the algorithm are given in Table 1.

It is assumed that the accelerations of the mobile nodes $u_{x}$ and $u_{y}$ can change within the range $[-5,5]\left[\mathrm{m} / \mathrm{s}^{2}\right]$ and the command process $u$ takes values among the following acceleration levels $\mathbb{M}=\left\{(0,0)^{\prime},(3.5,0)^{\prime},(0,3.5)^{\prime},(0,-3.5)^{\prime}\right.$, $\left.(-3.5,0)^{\prime}\right\}$. Thus, the number of motion modes is $r=5$. Nonrandom mobile node trajectories were generated with the dynamic state equations (1)-(3) without process noise. The initial state vectors are as follows: $\hat{x}_{1,0}=(-120,7,0,210$,

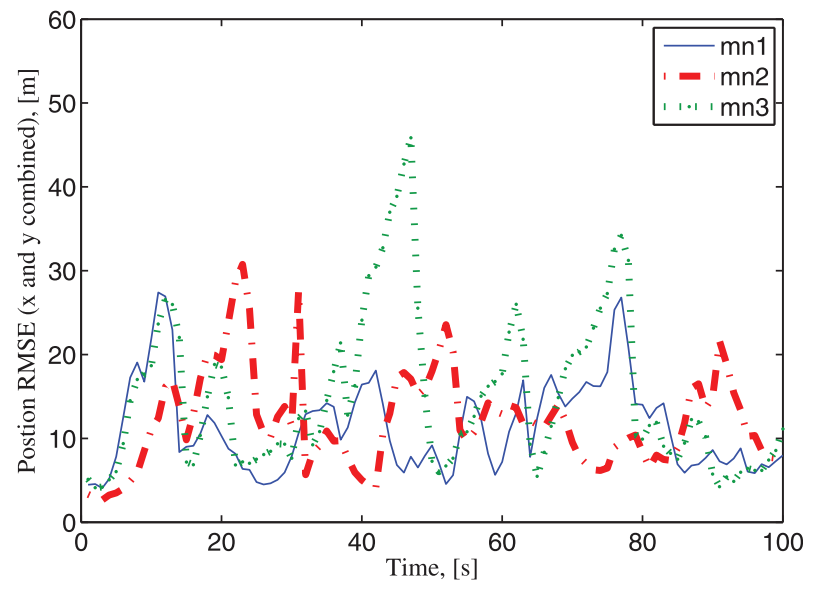

Fig. 4. Results for the speed RMSE obtained with the MM AUX-PF with an augmented state.

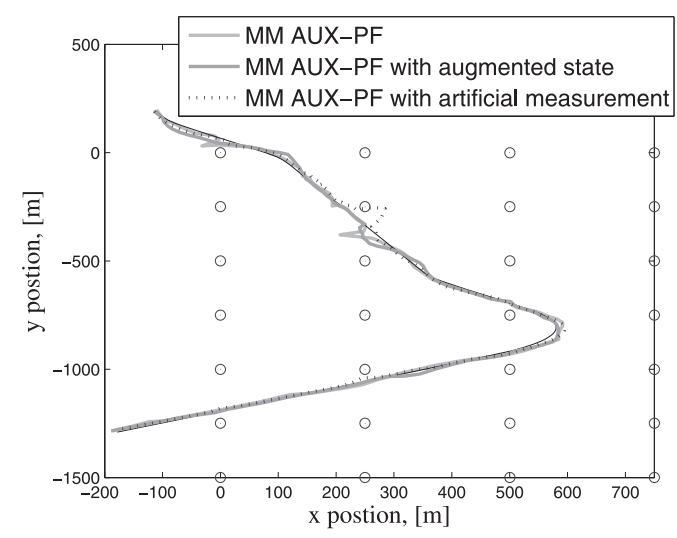

Fig. 5. Actual and estimated trajectories of the mobile node, obtained from: 1) the MM AUX-PF neglecting the temporal noise correlation, 2) an MM AUX-PF with augmented state vector, and 3) an MM AUX-PF with artificial measurement.

$-15,0)^{\prime}, \hat{x}_{2,0}=(550,7,0,150,-15,0)^{\prime}$, and $\hat{x}_{3,0}=(-630,7,0$, $350,-20,0)^{\prime}$. The first mobile node performs three short-term maneuvers with accelerations from the mode set and a longer maneuver with a control input $u=(-3.0,0.0)$, different from the acceleration set. The second mobile node maneuvers are described by the set of accelerations. The third mobile node performs two consecutive maneuvers with opposing accelerations. The root-mean-square errors (RMSE) [30] combined on both position coordinates yield the estimated state parameters to the actual dynamic parameters of each mobile node over $N_{m c}=50$ Monte Carlo runs. The position and speed RMSEs of the MM AUX-PF with augmented state are shown in Figs. 3 and 4. High position and speed estimates are achieved, with accuracy less than $45 \mathrm{~m}$ with respect to the mobile nodes position.

\subsubsection{Testing Scenario 2}

In this example, we perform a comparison between the developed MM AUX-PF with an augmented state vector and MM AUX-PF AM for dealing with the measurement time correlation and an MM AUX-PF without accounting for the measurement correlation. The estimated and actual trajectories are given in Fig. 5. The position and speed RMSEs are presented in Figs. 6 and 7, respectively, and show that the MM AUX-PF with the AS outperforms the MM AUX-PF neglecting the measurement noise correlation. 


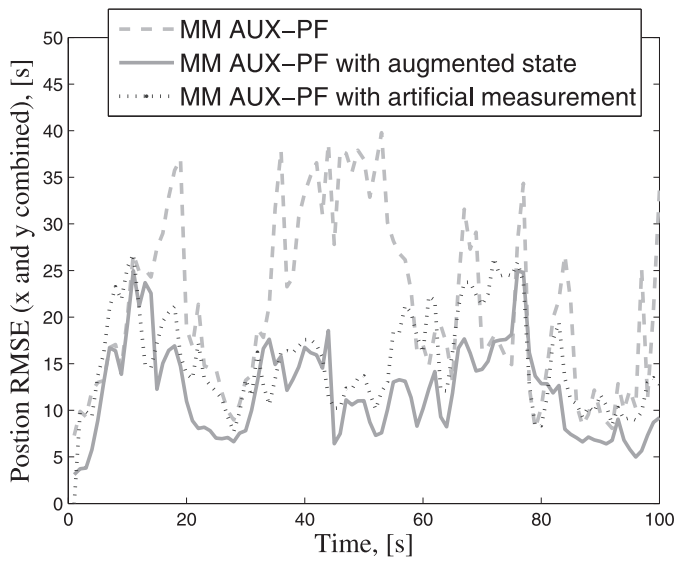

Fig. 6. Position RMSE (from 50 Monte Carlo runs): comparison between the MM AUX-PF (without taking into account the noise correlation), the MM AUX-PF with an augmented state vector, and the MM AUX-PF with artificial measurement.

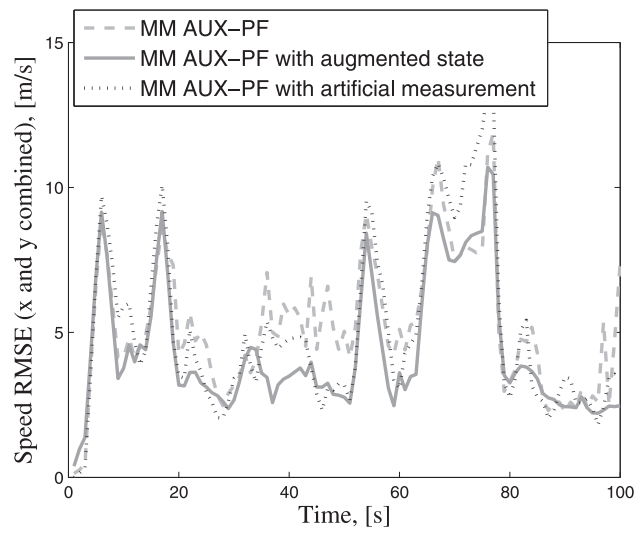

Fig. 7. Speed RMSE of the mobile node (from 50 Monte Carlo runs): between the MM AUX-PF (without taking into account the noise correlation)), the MM AUX-PF with an augmented state vector, and the MM AUX-PF with artificial measurement.

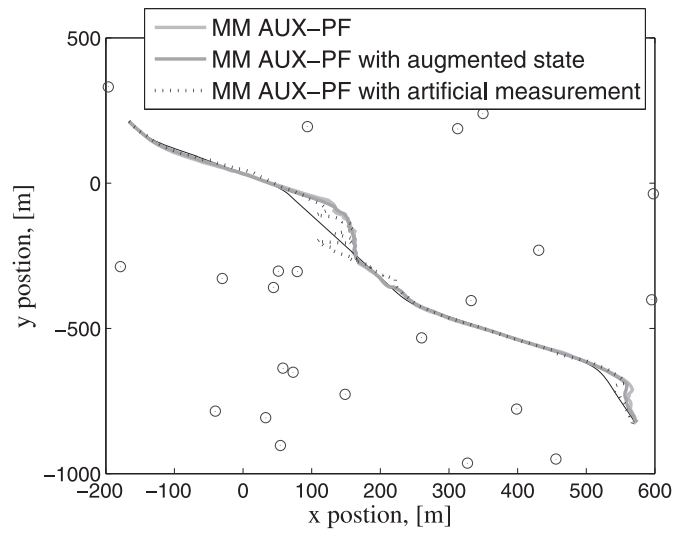

Fig. 8. Actual and estimated trajectory. Comparison between the MM AUX-PF, MM AUX-PF with an augmented state vector, and MM AUXPF with artificial measurement when sensors are randomly deployed.

The accuracy with respect to position is below 40 meters and the difference in the accuracy of the two filters is especially noticeable in the maneuvering stages.

\subsubsection{Testing Scenario 3}

This experiment shows a comparison between the performance of the MM AUX-PF AS and MM AUX-PF AM with the MM AUX-PF, neglecting the measurement correlation

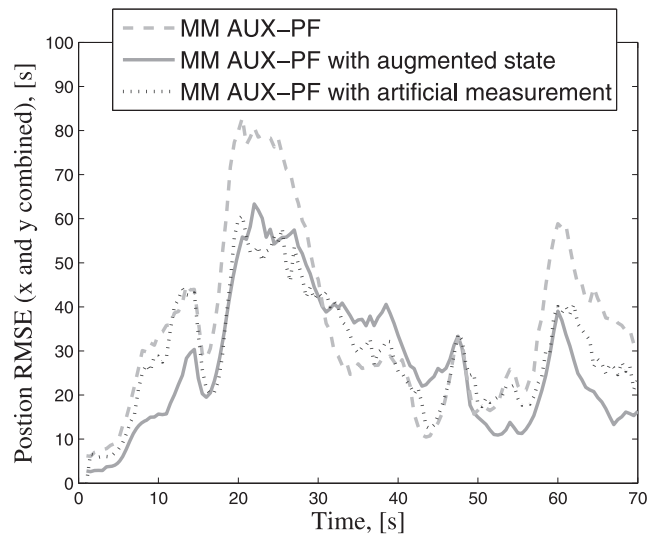

Fig. 9. Position RMSE of the mobile node: comparison between the MM AUX-PF, MM AUX-PF with an augmented state vector, and MM AUXPF with artificial measurement when sensors are randomly deployed.

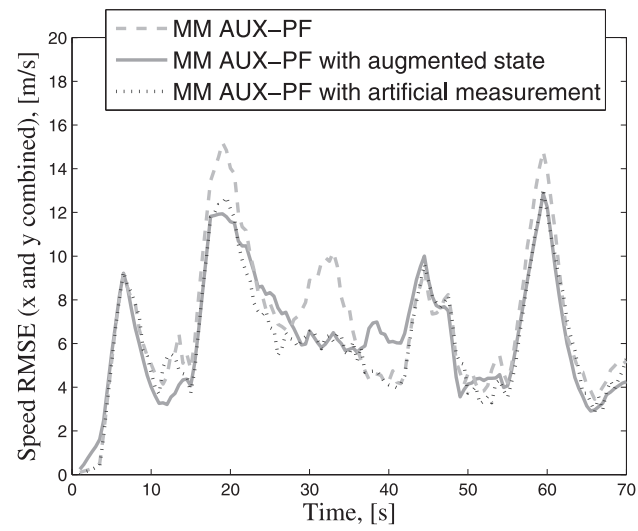

Fig. 10. Speed RMSE of the mobile node: comparison between the MM AUX-PF, MM AUX-PF with an augmented state vector, and MM AUXPF with artificial measurement when sensors are randomly deployed.

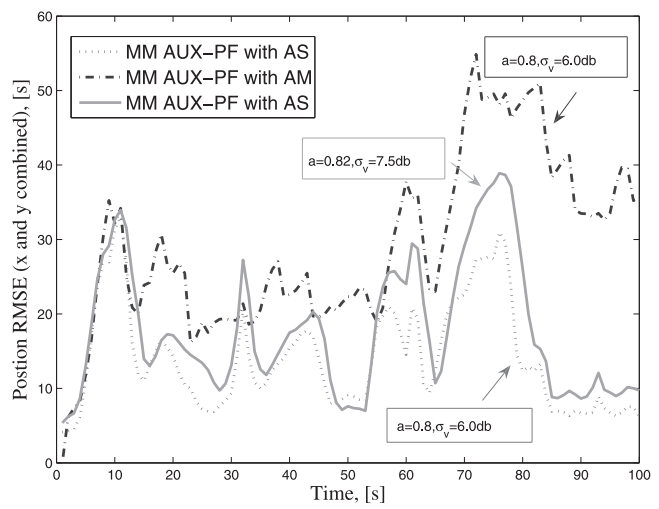

Fig. 11. Experiment with high values of the correlation coefficient (suburban environment). Position RMSE of the mobile node: comparison between the MM AUX-PF AS and the MM AUX-PF AM.

over a network of randomly deployed sensors. The results are presented in Figs. 8, 9, and 10.

Experiments were also performed with a higher value of the correlation coefficient 0.8 , higher shadowing noise $6^{2} \mathrm{~dB}$, and, respectively, 0.82 and $7.5^{2} \mathrm{~dB}$. The RMSE position error is shown in Fig. 11. From all the experiments, we can conclude that with a small correlation coefficient, the two MM AUX-PFs (both with AS and AM) outperform the MM AUX-PF neglecting the temporal correlation and the benefit is the biggest in the maneuvering periods. With high values 


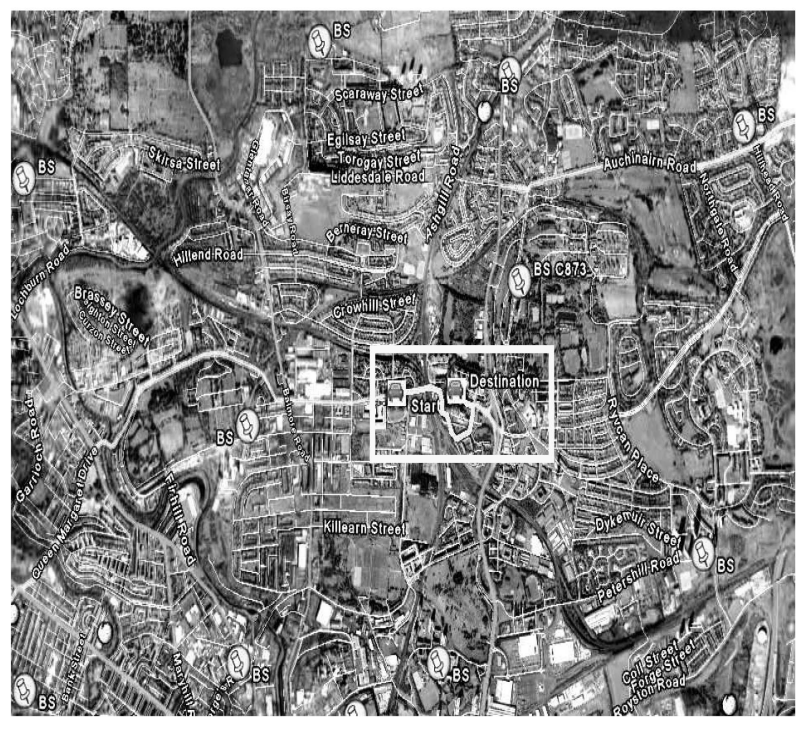

Fig. 12. The area in Glasgow, United Kingdom, where the vehicle is moving. The nearest BSs, the start, and destination positions are indicated on the map.

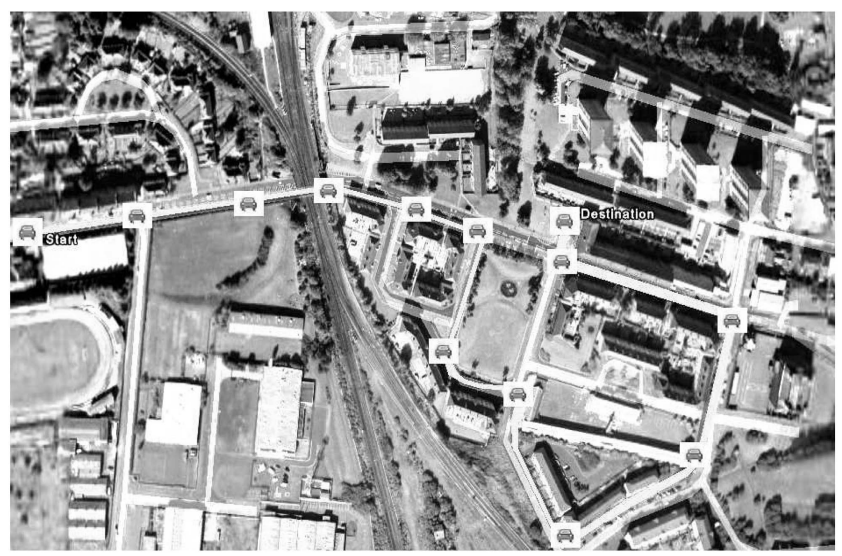

Fig. 13. The vehicle trajectory (from the left to the right side). The start and destination positions are indicated on the map.

of the correlation coefficient (e.g., 0.8), the algorithm neglecting the noise correlation is almost always divergent, whereas the MM AUX-PF (both with AS and AM) shows reliable performance. The MM AUX-PF AS has shown more accurate performance than the MM AUX-PF AM.

\subsection{Results with Real Data}

The performance of the proposed localization MM-AUX-PF algorithms, with AS and AM, respectively, has been investigated over a cellular wireless network, with real RSSIs, collected from BSs in Glasgow, United Kingdom. The mobile station was a vehicle driving in the city center. More than 400 BSs are available in the area where the car was moving. However, only data from the six with the highest RSSIs were provided to the localization algorithm. Also, a GPS system collected the actual positions of the moving mobile, for the purposes of validating the performance of the developed algorithms. Fig. 12 presents the map of the urban environment, with the nearest base stations and the trajectory of the car (shown increased in size in Fig. 13). The vehicle trajectory contains both patterns with sharp

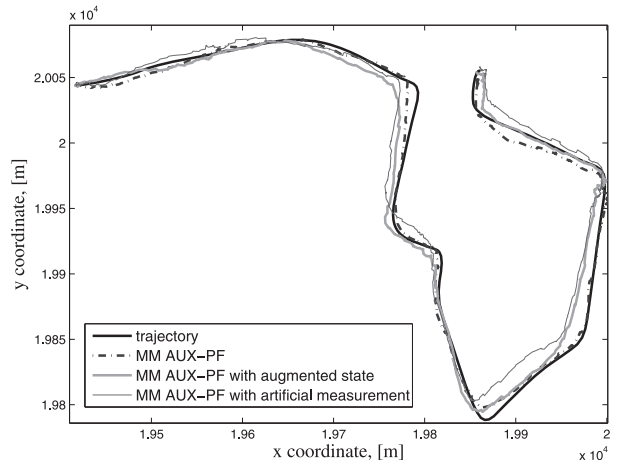

Fig. 14. This figure shows the actual and the estimated trajectory of the vehicle by: 1) the MM AUX-PF neglecting the measurement noise correlation, 2) the MM AUX-PF with augmented state, and 3) the MM AUX-PF with artificial measurement.

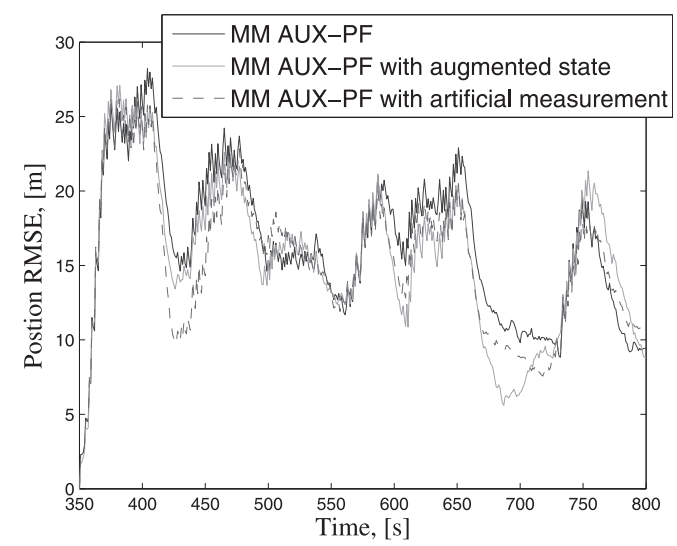

Fig. 15. Position RMSE for: 1) the MM AUX-PF neglecting the measurement noise correlation, 2) the MM AUX-PF with augmented state, and 3) the MM AUX-PF with artificial measurement.

maneuvers and rectilinear motion, including a stretch at the end where the vehicle is parked. Additional information for the road is included as position constraints in the algorithms.

Apart from the signal strengths, a GPS system collected the actual positions of the mobile unit. All parameters for the filters (tested with the real data) are the same as the parameters given in Table 1, except for the sampling period $T=0.5 \mathrm{~s}, V_{\max }=25 \mathrm{~m} / \mathrm{sec}$, sample size $T_{m}=800$, $\sigma_{x}=[30,1,0.3,30,1,0.3]^{\prime}$, and $\sigma_{v}=5.5 \mathrm{~dB}$. The transition probability matrix $\Pi$ has the same form as in Section 4.1. Fig. 14 shows the actual trajectory of the mobile together with the estimated trajectories, and Fig. 15 gives the respective position RMSEs. With the real RSSIs, we compared the performance of: 1) the MM AUX-PF that does not take into account the temporal measurement noise correlation with the MM AUX-PF with AM and MM AUX-PF with AS. From both Figs. 14 and 15, it is evident that the accuracy of the MM AUX-PF with AS and the MM-AUX PF with AM is higher than the accuracy of the MM AUX-PF neglecting the temporal noise correlation.

The estimated trajectory obtained with the MM AUX$\mathrm{PF}$ accounting for the noise correlation is more accurate than the MM AUX-PF estimate without taking into account this correlation.

The computational complexity is another important issue that we investigated. The MM AUX-PF execution time increases with the number of maneuvering models used in 
the implementation. The ratio between the computational time of the MM AUX-PF with five models and the computational time of the conventional AUX-PF is approximately 3:1. In the framework of the MATLAB environment, one-step processing time of a mobile node is approximately 2 seconds on a conventional PC (AMD Athlon(tm) 64 Processor $1.81 \mathrm{GHz}$ ). By using $\mathrm{C}++$ programming tools, the computational time is reduced to the sampling interval. In MATLAB environment with a nonoptimized code, the execution time for the MM-AUX PF with AS and AM is 2.33 and $2.38 \mathrm{~s}$, respectively.

\section{Conclusions}

This paper contributes to solving the problem of simultaneous localization of mobile nodes in wireless networks with correlated in time measurement noises. Two MM auxiliary particle filters (with an augmented state vector and an artificial measurement, respectively) are proposed for simultaneous localization of a mobile nodes in wireless networks. The algorithms performance has been investigated and validated over different scenarios and has shown high accuracy for localizing maneuvering nodes.

The developed techniques have the potential to be used in different applications, such as GPS-free position localization of mobile nodes in wireless networks, for localization of moving vehicles and robots. The algorithms proposed here can be useful also in scenarios where the location information for the mobile nodes is supporting basic network functions.

Future work will be focused on localization when both fixed and mobile nodes communicate with each other, on techniques for localizing of a large number of nodes and connectivity issues.

\section{ACKNOWLEDGMENTS}

The authors are thankful for the anonymous reviewers helping them to improve this paper. They acknowledge the support of EPSRC project EP/E027253/1, the (European Community's) Seventh Framework Programme (FP7/20072013) under grant agreement No. 238710 (Monte-Carlobased Innovative Management and Processing for an Unrivalled Leap in Sensor Exploitation), and the Bulgarian National Science Fund grant DTK02-28/2009. They are also grateful to HW Communications, Ltd., United Kingdom, for providing them with the real data.

\section{REFERENCES}

[1] M. Çetin, L. Chen, J. Fisher, A. Ihler, III, M. Wainwright, and A. Willsky, "Distributed Fusion in Sensor Networks," IEEE Signal Processing Magazine, vol. 23, no. 4, pp. 42-55, Dec. 2006.

[2] F. Zhao, J. Shin, and J. Reich, "Information-Driven Dynamic Sensor Collaboration for Tracking Applications," IEEE Signal Processing Magazine, vol. 21, no. 2, pp. 61-72, Mar. 2002.

[3] J.J. Xiao, A. Ribeiro, and Z.-Q. Luo, "Distributed CompressionEstimation Using Wireless Sensor Networks," IEEE Signal Processing Magazine, vol. 23, no. 4, pp. 27-41, July 2006.

[4] M. Mauve, J. Widmer, and H. Hartenstein, "A Survey on PositionBased Routing in Mobile Ad Hoc Networks," IEEE Network Magazine, vol. 15, no. 6, pp. 30-39, Nov./Dec. 2001.
[5] M. Srivastava, R. Muntz, M. Potkonjak, and S. Kindergarten, "Sensor-Based Wireless Networks for Smart Developmental Problem-Solving Environments," Proc. ACM SIGMOBILE, 2005.

[6] F. Gustafsson and F. Gunnarsson, "Mobile Positioning Using Wireless Networks: Possibilities and Fundamental Limitations Based on Available Wireless Network Measurements," IEEE Signal Processing Magazine, vol. 22, no. 4, pp. 41-53, July 2005.

[7] N. Patwari, J. Ash, S. Kyperountas, A. Hero, III, R. Moses, and N. Correal, "Locating the Nodes: Cooperative Localization in Wireless Sensor Networks," IEEE Signal Processing Magazine, vol. 22, no. 4, pp. 54-69, July 2005.

[8] R. Moses, D. Krishnamurthy, and R. Patterson, "A Self-Localization Method for Wireless Sensor Networks," EURASIP J. Applied Signal Processing, vol. 4, pp. 348-358, 2003.

[9] P. Juang, H. Oki, Y. Wang, L.S. Peh, and D. Rubinstein, "EnergyEfficient Computing for Wildlife Tracking: Design Tradeoffs and Early Experiences with ZebraNet," Proc. Conf. Architectural Support for Programming Languages and Operating Systems, pp. 96-107, 2002.

[10] P. Djurić, M. Vemula, M. Bugallo, and J. Miguez, "NonCooperative Localization of Binary Sensors," Proc. IEEE Statistical Signal Processing Workshop, 2005.

[11] G. Sun, J. Chen, W. Guo, and K. Liu, "Signal Processing Techniques in Network-Aided Positioning: A Survey of State-ofthe-Art Positioning Designs," IEEE Signal Processing Magazine, vol. 22, no. 4, pp. 12-23, July 2005.

[12] K. Chintalapudi, A. Dhariwal, R. Govindan, and G. Sukhatme, "Ad-Hoc Localization Using Ranging and Sectoring," Proc. IEEE INFOCOM, 2004.

[13] F. Gustafsson and F. Gunnarsson, "Localization in Sensor Networks Based on Log Range Observations," Proc. 10th Int'l Conf. Information Fusion, 2007.

[14] J. Jirod and D. Estrin, "Robust Range Estimation Using Acoustic and Multimodal Sensing," Proc. IEEE Int'l Conf. Intelligent Robots and Systems, 2001.

[15] T. He, C. Huang, B.M. Blum, J.A. Stankovic, and T. Abdelzaher, "Range-Free Localization Schemes for Large Scale Sensor Networks," Proc. MobiCom, pp. 81-95, 2003.

[16] Z. Zaidi and B. Mark, "A Mobility Tracking Model for Wireless Ad Hoc Networks," Proc. IEEE Wireless Comm. and Networking Conf. (WCNC '03), vol. 3, pp. 1790-1795, 2003.

[17] Z. Zaidi and B. Mark, "Mobility Estimation for Wireless Networks Based on an Autoregressive Model," Proc. IEEE Global Telecomm. Conf. (Globecom), pp. 3405-3409, 2004.

[18] L. Hu and D. Evans, "Localization for Mobile Sensor Networks," Proc. 10th Ann. Int'l Conf. Mobile Computing and Networking, 2004.

[19] L. Mihaylova, D. Angelova, C.N. Canagarajah, and D.R. Bull, "Algorithms for Mobile Nodes Self-Localisation in Wireless Ad Hoc Networks," Proc. Ninth Int'l Conf. Information Fusion, 2006.

[20] A. Ihler, J. Fisher, R. Moses, and A. Willsky, "Nonparametric Belief Propagation for Sensor Network," IEEE J. Selected Areas in Comm., vol. 23, no. 4, pp. 809-819, Apr. 2005.

[21] L. Mihaylova, D. Bull, D. Angelova, and N. Canagarajah, "Mobility Tracking in Cellular Networks with Sequential Monte Carlo Filters," Proc. Eight Int'l Conf. Information Fusion, 2005.

[22] L. Mihaylova, D. Angelova, S. Honary, D.R. Bull, C.N. Canagarajah, and B. Ristic, "Mobility Tracking in Cellular Networks Using Particle Filtering," IEEE Trans. Wireless Comm., vol. 6, no. 10, pp. 3589-3599, Oct. 2007.

[23] R. Karlsson and N. Bergman, "Auxiliary Particle Filters for Tracking a Manoeuvring Target," Proc. 39th IEEE Conf. Decision and Control, pp. 3891-3895, 2000.

[24] M.S. Arulampalam, B. Ristic, N. Gordon, and T. Mansell, "Bearings Only Tracking of Manoeuvring Targets Using Particle Filters," EURASIP J. Applied Signal Processing, vol. 2004, no. 1, pp. 2351-2365, 2004.

[25] P.M. Djuric, M. Vemula, and M.F. Bugallo, "Target Tracking by Particle Filtering in Binary Sensor Networks," IEEE Trans. Signal Processing, vol. 56, no. 6, pp. 2229-2238, June 2008.

[26] A. Kannan, G. Mao, and B. Vucetic, "Simulated Annealing Based Wireless Sensor Network Localization with Flip Ambiguity Mitigation," Proc. IEEE Vehicular Technology Conf. Spring (VTC), pp. 1022-1026, 2006.

[27] M. Gudmundson, "Correlation Model for Shadow Fading in Mobile Radio Systems," Electronics Letters, vol. 27, no. 23, pp. 21452146, 1991. 
[28] T. Jiang, N. Sidiropoulos, and G. Giannakis, "Kalman Filtering for Power Estimation in Mobile Communications," IEEE Trans. Wireless Comm., vol. 2, no. 1, pp. 151-161, Jan. 2003.

[29] I. Forkel, M. Schinnenburg, and M. Ang, "Generation of Two Dimensional Correlated Shadowing for Mobile Radio Network Simulation," Proc. Seventh Int'l Symp. Wireless Personal Multimedia Comm., pp. 314-319, Sept. 2004.

[30] Y. Bar-Shalom, X.R. Li, and T. Kirubarajan, Estimation with Applications to Tracking and Navigation. John Wiley and Sons, 2001.

[31] T. Camp, J. Boleng, and V. Davies, "A Survey of Mobility Models for Ad Hoc Network Research," Wireless Comm. and Mobile Computing, vol. 2, no. 5, pp. 483-502, Aug. 2002.

[32] B. Mark and Z. Zaidi, "Robust Mobility Tracking for Cellular Networks," Proc. IEEE Int'l Comm. Conf., pp. 445-449, May 2002.

[33] Z.R. Zaidi and B.L. Mark, "Real-Time Mobility Tracking Algorithms for Cellular Networks Based on Kalman Filtering," IEEE Trans. Mobile Computing, vol. 4, no. 2, pp. 195-208, Mar./Apr. 2005.

[34] R. Moose, "An Adaptive State Estimator Solution to the Maneuvering Target Tracking Problem," IEEE Trans. Automatic Control, vol. 20, no. 3, pp. 359-362, June 1975.

[35] Y. Bar-Shalom and X. Li, Estimation and Tracking: Principles, Techniques and Software. Artech House, 1993.

[36] X.R. Li and V. Jilkov, "A Survey of Maneuvering Target Tracking. Part I: Dynamic Models," IEEE Trans. Aerospace and Electronic Systems, vol. 39, no. 4, pp. 1333-1364, Oct. 2003

[37] Z. Yang and X. Wang, "Joint Mobility Tracking and Hard Handoff in Cellular Networks via Sequential Monte Carlo Filtering," Proc. IEEE INFOCOM, 2002.

[38] Z. Yang and X. Wang, "Sequential Monte Carlo for Mobility Management in Wireless Cellular Networks," Proc. XI European Signal Processing Conf. (EUSIPCO), 2002.

[39] G. Stüber, Principles of Mobile Communication, second ed. Kluwer Academic Publisher, 2001.

[40] L. Hissalle and S. Alahakoon, "Estimating Signal Strengths Prior to Field Trials in Wireless Local Loop Networks," Proc. Int'l Conf. Industrial and Information Systems, pp. 409-414, Aug. 2007.

[41] M. Arulampalam, S. Maskell, N. Gordon, and T. Clapp, "A Tutorial on Particle Filters for Online Nonlinear/Non-Gaussian Bayesian Tracking," IEEE Trans. Signal Processing, vol. 50, no. 2, pp. 174-188, Feb. 2002.

[42] J. Liu and R. Chen, "Sequential Monte Carlo Methods for Dynamic Systems," J. Am. Statistical Assoc., vol. 93, no. 443, pp. 1032-1044, 1998

[43] E. Wan and R. van der Merwe, "The Unscented Kalman Filter," Kalman Filtering and Neural Networks, S. Haykin, ed., chapter 7, pp. 221-280, Wiley, Sept. 2001.

[44] M.K. Pitt and N. Shephard, "Filtering via Simulation: Auxiliary Particle Filters," J. Am. Statistical Assoc., vol. 94, no. 446, pp. 590$599,1999$.

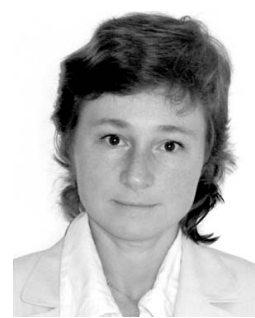

Lyudmila Mihaylova is a Reader in Advanced Signal Processing with the School of Computing and Communications, Lancaster University, United Kingdom. She has published more than 80 papers in leading scientific journals and peerreviewed international conference proceedings. Her interests are in the area of nonlinear filtering, statistical signal and image processing, autonomous intelligent systems, and sensor data fusion. She is the editor-in-chief of the Open Transportation Journal and an associate editor of the Elsevier's Signal Processing journal. She is the chair of a work group on "Multi-Sensor Data Fusion of Traffic and Weather Data" and the EU Management Committee of EU COST action: "Real-Time Monitoring, Surveillance and Control of Road Networks under Adverse Weather Conditions" TU0702 (2007-2010). Her research is supported by the EU, EPSRC, and industry. She is a senior member of the IEEE. More details about her research can be found at http://www.lancs.ac.uk/staff/mihaylov.

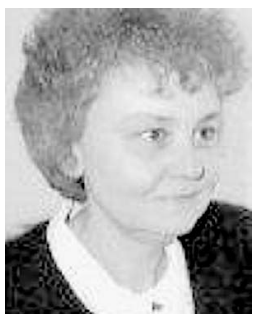

Donka Angelova received the MS degree in mechanical engineering from the Technical University of Sofia, Bulgaria, and the $\mathrm{PhD}$ degree in electrical engineering from the Bulgarian Academy of Sciences, Institute for Parallel Processing, where she is an academic rank associate research professor. She works as a key researcher and is involved in academic and industry projects (Bulgarian and international) in the areas of multitarget tracking and multisensor data fusion. Her current research interests include the application of sequential Monte Carlo methods to the problems of applied estimation and filtering and decision making.

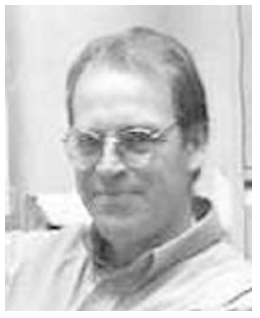

David R. Bull received the BSc degree form the University of Exeter in 1980, the MSc degree from the University of Manchester in 1983, and the $\mathrm{PhD}$ degree from the University of Cardiff in 1988. He currently holds the chair in signal processing at the University of Bristol, where he was the head of the Electrical and Electronic Engineering Department between 2001 and 2006 and is now the director of the Bristol Vision Institute (BVI). His previous roles include being a lecturer at the University of Wales, Cardiff, and a systems engineer for Rolls Royce. In 2001, he cofounded ProVision Communication Technologies, Ltd., where he is now the chairman. He has worked widely in the fields of $1 \mathrm{D}$ and $2 \mathrm{D}$ signal processing. He has won two IEE Premium awards for this work and has published numerous patents, several of which have been exploited commercially. His current activities are focused on the problems of image and video communications and analysis for low bit rate wireless, Internet, military, and broadcast applications. He has published more than 370 academic papers, various articles, and two books, and has also given numerous invited/keynote lectures and tutorials. He is a senior member of the IEEE.

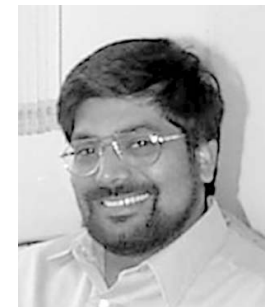

Nishan Canagarajah received the BA (Hons) and $\mathrm{PhD}$ degrees in DSP techniques for speech enhancement from the University of Cambridge. $\mathrm{He}$ is a professor of multimedia signal processing at Bristol, United Kingdom. Prior to this, he was a research assistant and a lecturer at Bristol, investigating digital signal processing (DSP) aspects of mobile radio receivers. His research interests include image and video coding, image segmentation, content-based video retrieval, 3D video, image fusion, and the application of signal processing to audio and medical electronics. He is widely supported in these areas by industry, EU, and the EPSRC. He has been involved in a number of FP5, FP6, and F7 projects where the team has been developing novel image/video processing algorithms. He has published more than 150 papers and two books. He is a member of the EPSRC College and the IEEE.

$\triangleright$ For more information on this or any other computing topic, please visit our Digital Library at www.computer.org/publications/dlib. 